\title{
Scaling Structures in Four-dimensional Simplicial Gravity *
}

\author{
H.S.Egawa ${ }^{a}$, T.Hotta ${ }^{b}$, T.Izubuchi ${ }^{b}$ N.Tsuda ${ }^{c}$ and T.Yukawa ${ }^{c}, d$ \\ ${ }^{a}$ Department of Physics, Tokai University Hiratsuka, Kanagawa 259-12, Japan \\ b Department of Physics, University of Tokyo Bunkyo-ku, Tokyo 113, Japan

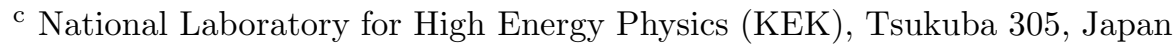 \\ d Coordination Center for Research and Education, The Graduate University for Advanced Studies, \\ Hayama-cho, Miura-gun, Kanagawa 240-01, Japan
}

Four-dimensional(4D) spacetime structures are investigated using the concept of the geodesic distance in the simplicial quantum gravity. On the analogy of the loop length distribution in $2 \mathrm{D}$ case, the scaling relations of the boundary volume distribution in $4 \mathrm{D}$ are discussed in various coupling regions i.e. strong-coupling phase, critical point and weak-coupling phase. In each phase the different scaling relations are found.

\section{Introduction}

Simplicial gravity has witnessed a remarkable development toward quantizing the Einstein gravity. This development started with $2 \mathrm{D}$ simplicial gravity and has now reached the point of subjecting to simulate $4 \mathrm{D}$ case 1 , 4 about the analysis of fractal dimensions, minbu, scaling relations for the loop length distribution and the curvature distribution. The aim of this paper is to investigate 4D Euclidean spacetime structures using the concept of the geodesic distance. It is very important that the scaling relations have been obtained in $2 \mathrm{D}$ case. Therefore, on the analogy of the loop length distribution(LLD) in 2D [5], the scaling relations in $4 \mathrm{D}$ are discussed. Actually we measured the boundary volume distribution(BVD) for various geodesic distances in $4 \mathrm{D}$ dynamically triangulated(DT) manifold, in analogy to LLD. In order to discuss the scaling relations, we assume that the scaling variable $x$ has a form $x=V / D^{\alpha}$, where $V, D$ and $\alpha$ denote the each boundary(cross section) volume, the geodesic distance and scaling parameter, respectively. Hagura et al. argue the scaling properties of the surface area distributions in 3D case by the same analysis as we employ in $4 \mathrm{D}$ case(in these proceedings).

*presented by H.S.Egawa

\section{The model}

We use the lattice action of $4 \mathrm{D}$ model with the $S^{4}$ topology corresponding to the action as $S=-\kappa_{2} N_{2}+\kappa_{4} N_{4}$, where $N_{i}$ denotes the total number of $i$-simplexes. The coupling $\kappa_{2}$ is proportional to the inverse bare Newton constant and the coupling $\kappa_{4}$ corresponds to a lattice cosmological constant. For the dynamical triangulation model of $4 \mathrm{D}$ quantum gravity, we consider a partition function of the form, $Z\left(\kappa_{2}, \kappa_{4}\right)=$ $\sum_{T\left(S^{4}\right)} e^{-S\left(\kappa_{2}, \kappa_{4}, T\right)}$. We sum up over all simplicial triangulations $T\left(S^{4}\right)$. In practice, we have to add a small correction term, $\Delta S=\delta \kappa_{4}\left(N_{4}-\right.$ $\left.N_{4}^{(\text {target })}\right)^{2}$, to the action in order to suppress the volume fluctuations from the target value of 4 simplexes $N_{4}^{(\text {target })}$ and we have used $\delta=0.0005$.

\section{Numerical Simulations and Results}

We define $N_{b}(D)$ as the number of boundaries at the geodesic distance $D$ from a reference 4-simplex in the 4D DT manifold averaged over all 4-simplexes. Fig.11 shows the distributions of $N_{b}(D)$ for the typical three coupling strength with $N_{4}=16 \mathrm{~K}$. In the strong coupling limit $\left(\kappa_{2}=0\right)$, the only one boundary that is identified as the mother universe exists almost all the distances(see Fig.1), which means 


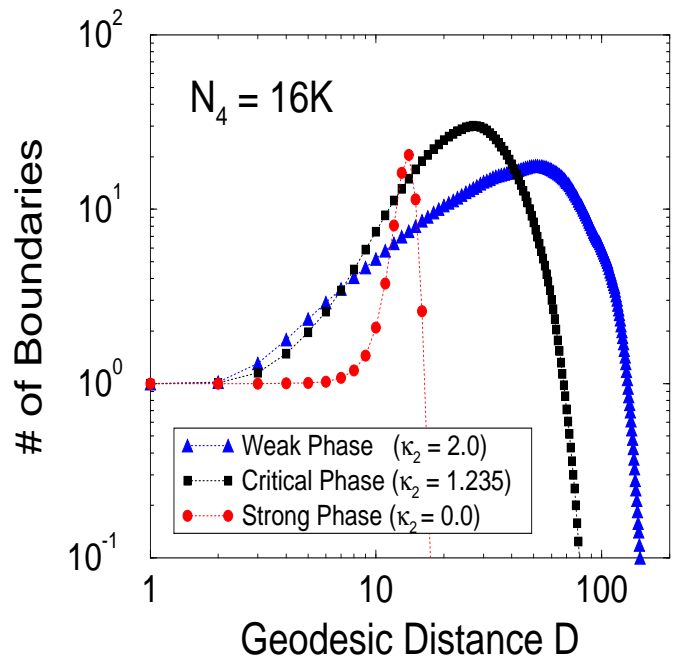

Figure 1. Number of Boundaries plotted versus geodesic distances $D$, with the double-log scales.

that the mother universe is a dominant structure. The branching structures are highly suppressed, which shows characteristic properties of the "crumpled manifold", which is similar to the case observed in $2 D$ manifold. On the other hand, in the weak coupling phase(for example, we chose $\kappa_{2}=2.0$ ), we observe the growth of the branches until $D \sim 60$ and can reasonably extract the relation $N_{b}(D) \propto D$ in the region $3 \leq D \leq 30$. Then we call this manifold as the "elongated manifold".

\subsection{The strong coupling phase}

Fig.2 shows BVD, $\rho(x)$, with $x=V / D^{4.5}$ as a scaling variable in the strong coupling region, $\kappa_{2}=0$, with $N_{4}=32 K$ while the fractal dimension $\left(d_{f}\right)$ reaches about 5.5 which yet increases with the volume in our simulation size. In terms of this variable the mother universe shows scaling relation and distributes like a Gaussian distribution. We can be fairly certain that in the strong coupling phase the scaling parameter $\alpha$ of mother universe satisfies the relation $d_{f}=\alpha+1$, and the manifold resembles a $d_{f}$-sphere $\left(S^{d_{f}}\right)$. There seems to be a scaling property with respect to BVD with $x=V / D^{d_{f}-1}$ as a scaling variable.

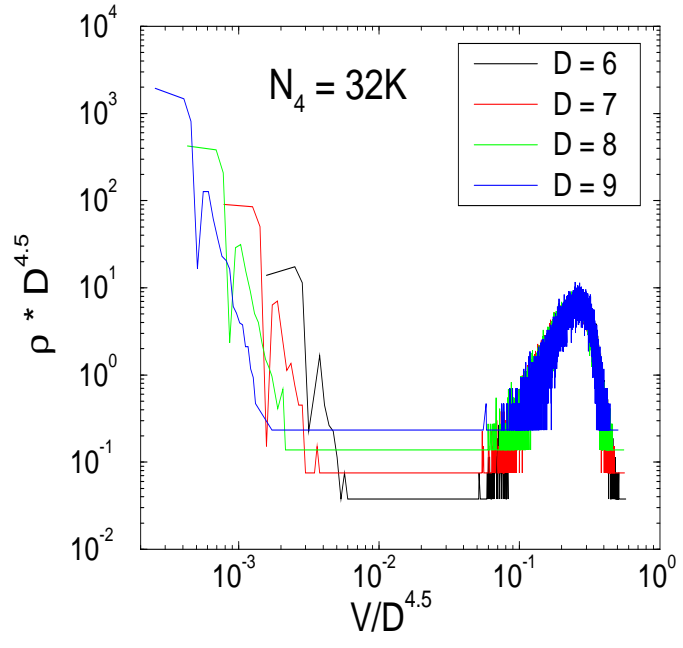

Figure 2. BVD plotted versus a scaling variable $x=V / D^{4.5}$, with the double-log scales in the strong coupling limit: $\kappa_{2}=0$ and $N_{4}=32 K$.

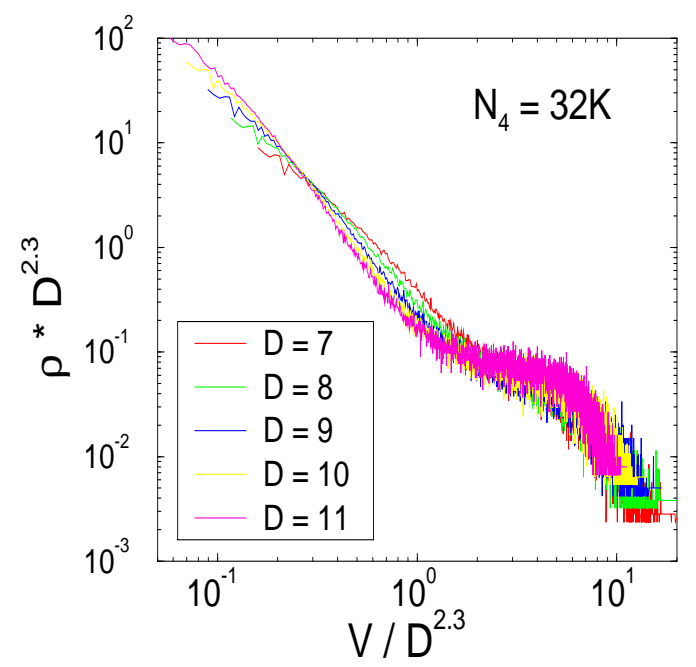

Figure 3. BVD plotted versus a scaling variable $x=V / D^{2.3}$, with the double-log scales at the critical point: $\kappa_{2}=1.2581$ and $N_{4}=32 K$. 


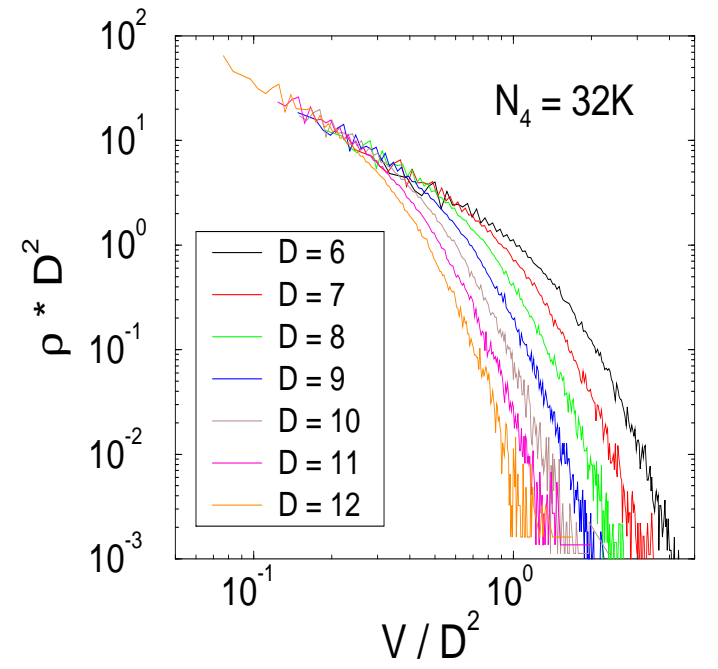

Figure 4. BVD plotted versus a scaling variable $x=V / D^{2}$, with the double-log scales in the weak coupling phase: $\kappa_{2}=2.0$ and $N_{4}=32 \mathrm{~K}$.

\subsection{The critical point}

Next, the data near the critical point are shown in Fig. 3 for various geodesic distances with $N_{4}=$ $32 K$. At this point the fractal dimension reaches about 3.5 and yet increasing for larger values of $N_{4}$. We must draw attention to the double peak structure on the critical point [6]. Therefore, we measure the boundary volume distribution on both peaks, and obtain the more clear signal of the distribution of the mother universe on the peak which is close to the strong coupling phase. We observe the scaling properties for the mother universe $\rho(x)$ with $x=V / D^{2.3}$ in Fig.3. In order to discuss the universality of the scaling relations, we assume the distribution function in terms of a scaling parameter $x=V / D^{2.3}$ as $\rho(x)=a_{0} \frac{1}{D^{2.3}} x^{a_{1}} e^{-a_{2} x}$, where $a_{0}, a_{1}$ and $a_{2}$ are some constants. Then we can calculate the fractal dimension from $\rho(x, D)$, $\lim _{x \rightarrow \infty} \int_{v_{0}}^{\infty} d V \quad V \quad \rho(x, D)=V^{(4)}(D) \sim D^{d_{f}}$, where $v_{0}$ denotes the cut-off volume and $V^{(4)}$ denotes the total volume of the $4 \mathrm{D}$ manifold. If $a_{1}>-2$ this integration is convergent and gives a finite fractal dimension. We can extract the func- tion of $\rho(x, D)$ from Fig. 3 , and find $a_{1} \simeq 0.5$ for the distribution of mother volume and $a_{2} \simeq 3.0$. Furthermore we investigate the $N_{4}=64 K$ case and obtain the same scaling behavior as that of $N_{4}=32 K$ case except $d_{f} \simeq 4.0$ and scaling parameter $\alpha \simeq 3.0$. These results lead to the conclusion that the distributions of the baby universes show no scaling behavior. On the other hand, the distribution of the mother universe shows the scaling relation and is universal(i.e. it does not depend on the lattice cut-off $\left.\left(v_{0}\right)\right)$.

\subsection{The weak coupling phase}

Finally, we show the data in the weak coupling phase $\left(\kappa_{2}=2.0\right)$ within which the fractal dimension reaches about 2.0. Fig. 1 shows BVD with $x=V / D^{2}$ as a scaling variable. We can safely state $\rho(x) \times D^{2} \propto x^{-2.0} e^{-x}$. In this phase, the dynamically triangulated manifold consists of widely expanding like branched polymers and we cannot observe the mother universe at all.

\section{Summary and discussions}

On the analogy of LLD in 2D case, the scaling relations in $4 \mathrm{D}$ are discussed for the three phases. BVD, $\rho(x, D)$, at geodesic distance $D$ gives us some basic scaling relations on the ensemble of Euclidean space-times described by the partition function $Z\left(\kappa_{2}, \kappa_{4}\right)$.

In the strong coupling limit $\kappa_{2}=0$ we find that the mother part of BVD, $\rho(x, D)$, scales trivially with $x=V / D^{d_{f}-1}$ as a scaling variable. There is fairly general agreement that the 4D DT manifold seems to be a $d_{f}$-sphere $\left(S^{d_{f}}\right)$. What is important is that this scaling property for the mother universe changes gradually into the scaling relation of that of the critical point. The fluctuations of the spacetime growth with $\kappa_{2} \rightarrow \kappa_{2}^{c}$. In $2 \mathrm{D}$, the baby loop and the mother loop show scalings with the same parameter $\left(x=L / D^{2}\right)$. However, LLD of the baby loops is depend on the lattice cutoff and we think that it is not universal. At the critical point in $4 \mathrm{D}$ case we have obtained the similar BVD. However, we have a different scaling parameters $\left(x=V / D^{2.3}\right.$ with $N_{4}=32 K$ and $x=V / D^{3.0}$ with $\left.N_{4}=64 K\right)$ from $2 \mathrm{D}$ case for the mother universe. Furthermore, BVD of the 
baby universes seems to be non-universal.

In the weak coupling phase(see Fig.4) we have obtained the elongated manifolds, in other words, branched polymers. In this phase no mother universe exists and BVD of the baby universes shows that the scaling relation is not universal.

The results of this paper is the first step to research the universal scaling relations in 2, 3 and $4 \mathrm{D}$ on simplicial quantum gravity. The results of simulations can be regarded as the possibility that we may have $4 \mathrm{D}$ quantum gravity as the generalized DDK model.

-Acknowledgment-

We would like to thank H.Kawai, N.Ishibashi, S.R.Das, J.Nishimura and H.Hagura for fruitful discussions. Some of the authors (T.H., T.I. and N.T.) were supported by a Research Fellowships

of the Japan Society for the Promotion of Science for Young Scientists.

\section{REFERENCES}

1. M.E.Agishtein and A.A.Migdal, Nucl.Phys.B 385 (1992) 395.f

2. J.Ambjørn and J.Jurkiewicz, Nucl.Phys.B451 (1995) 643.

3. S.Catterall, J.Kogut and R.Renken, Phys.Lett.B 328 (1994) 277.

4. B.V.de Bakker and J.Smit, Nucl.Phys.B 439 (1995) 239.

5. H.Kawai, N.Kawamoto, T.Mogami and Y.Watabiki, Phys.Lett. B306 (1993) 19; N.Tsuda and T.Yukawa Phys.Lett. B305 (1993) 223.

6. P.Bialas, Z.Burda, A.Krzywicki and B.Petersson, hep-lat/9601024; B.V.de Bakker, hep-lat/9603024. 